\title{
Immediate Implantation and Prosthetic Rehabilitation
}

\author{
${ }^{1}$ Porus S Turner, ${ }^{2}$ Ferzin D Vazifdar, ${ }^{3}$ Ashdin P Turner, ${ }^{4}$ Danesh R Vazifdar, ${ }^{5}$ Rashnavi K \\ Masalawala, ${ }^{6}$ Bilquis J Ghadiali, ${ }^{7}$ Cyrus S Karkaria, ${ }^{8}$ Radhika B Parekh \\ ${ }^{1}$ Professor, Department of P rosthodontics, Mumbai, Maharashtra, India \\ ${ }^{2,3}$ Turner's Speciality Dental Center, Mumbai, Maharashtra, India \\ ${ }^{4}$ Ameretat Dental Labs, Mumbai, Maharashtra, India \\ 5-8Turner's Dental Care Center, Mumbai, Maharashtra, India
}

Correspondence: P orus S Turner, Professor, Department of Prosthodontics, AB Shetty Institute of Dental Sciences, Mangalore Karnataka, India, e-mail: porusturner@ hotmail.com

\begin{abstract}
Extraction and immediate implantation has become a very reliable and time saving method of prosthetic rehabilitation of a patient. Achieving high primary stability and preservation of hard and soft tissues is of great importance with this treatment modality. Therefore using implant system which addresses these concerns would be important. The presenter with his vast experience with oral implantology will highlight his own method of atraumatic extraction, implantation and immediate provisionalization with nonfunctional loading, with the help of clinically treated cases. He will demonstrate his method which may have universal acceptance.
\end{abstract}

Keywords: Immediate implant placement, optimum primary stability, nonfunctional loading, provisional restoration.

Implant placement immediately after extraction followed by an immediate provisional restoration can be a very rewarding way to provide implant therapy to our patients. It provides significant benefit both to the patient and the dentist compared with traditional delayed protocol of waiting for 6 months. It results in an improved preservation of the soft tissue drape and the bone architecture compared with their collapse after tooth extraction. In selected situations, it can even result in avoidance of bone augmentation and soft tissue grafts. The procedure also reduces the number of surgeries which may be beneficial both to the dentist and the patient (Table 1). Factors critical to success include good initial implant primary stability in good quality bone. It is generally avoided when the labial plate of bone is missing and grafting is required. However, immediate implant placement and loading is technically challenging and should only be undertaken by clinicians with considerable experience with implant dentistry, both surgically and prosthetically.

Table 1: Advantages of immediate implant placement into extraction sockets and provisional restorations

\footnotetext{
- Immediate esthetics by a fixed restoration

- Prevents collapse of the peri-implant soft tissue through support given by a carefully crafted provisional restoration

- Reduces the number of surgeries

- Reduces time required for rehabilitation.
}

\section{CRESTAL BONE LOSS AND THE BIOLOGIC WIDTH}

Preservation of crestal bone after 2 stage implant placement has been a concern. B ecause of the importance of bone to esthetics, the postoperative reduction of crestal bone after 2 stage implant therapy needs to be addressed. Crestal bone loss results in recession of the gingival margins and sometimes the papilla, particularly in individuals with thin biotypes.

The manner in which the junctional epithelium and connective tissue attaches to the tooth and to the implant differ. Whilst the junctional epithelium attaches by way of glycoproteins to the natural dentition, a pseudo attachment through hemidesmosomes exists around endosseous implants. In addition, connective tissue fibers mechanically insert into natural root cementum whereas in the case of implants, a tight cuff of connective tissue is formed around the titanium implant. ${ }^{1}$

Gargiulo ${ }^{2}$ documented a biologic width from the base of the gingival sulcus to the crest of the alveolar bone to be $2.04 \mathrm{~mm}$, with epithelial attachment of $0.97 \mathrm{~mm}$ and connective tissue attachment of $1.07 \mathrm{~mm}$. The dimensions in implants are supposed to be similar to those of natural teeth and are stable even after loading. These dimensions are a key determinant of esthetics. 
Following abutment connection, the crestal bone loss has been shown to recede from the implant abutment connection by 1.3 to $1.4 \mathrm{~mm}$ as measured horizontally. ${ }^{3}$ However, with certain implant systems like 'Ankylos' (Dentsply Friadent, Germany) which employ platform switching of the implant abutment connection, this crestal bone loss at Stage 2 surgery is nearly eliminated. The 'A nkylos' system in the mid 1980's was the first system to introduce the morse taper conical connection with platform switching of the implant abutment connection. Other companies who have followed in adapting this feature are A straZeneca, Sweden; B icon, B oston; Curasan, Germany and more recently Nobel A ctive, Nobel Biocare; and Straumann, Switzerland. The 'A nkylos' system is unique because it not only has platform switching which brings the bacterial inflammatory infiltrate from the bone towards the axis of the implant but more important the very strong conical implant abutment connection has no microgaps and also prevents micromovements resulting in no bacterial colonization. This unique combination of platform switching and strong implant abutment connection has been documented since the last 20 years to virtually eliminate crestal bone loss which in turn preserves soft tissue architecture..$^{4-7}$ The preservation of the perimplant bone is particularly important in the esthetic zone where the objective is to prevent postprosthetic bone loss and preserve soft tissues. The 'A nkylos' implant system is also grit blasted and thermally acid etched right up to the shoulder of the implant with no polished collar which encourage the bone to grow right up to the top of the implant thus allowing $1 \mathrm{~mm}$ subcrestal placement of the implant. Implants with smooth collars when placed subcrestally, the bone resorbs all the way down to the rough-to-smooth transition line.

Immediate placement and providing a customized immediate provisional restoration with appropriate subgingival emergence profile can be of significant benefit in providing stability to the periimplant soft tissues. Careful manipulations of the hard and soft tissues is critical to a successful outcome of the procedure. Extractions should be done as atraumatically as possible with microsurgical blades, periotomes and fine luxators taking care not to injure or fracture the labial cortical plate of bone. Intrasulcular incisions and minimal or no reflection of the periosteum (flapless procedure) should be employed.

Although, implant placement into fresh extraction sockets is now considered to be a predictable and accepted treatment procedure. There are important prerequisites for immediate implantation which must be addressed (Table 2).
Table 2: Prerequisites for immediate implantation

- Adequate primary stability

- Adequate bone all around the implant covering and stabilizing the implant

- Preferably no grafting procedure need to be undertaken especially no major grafting. If grafting has to be done, implant should be submerged wait for bone and implant to osseiointegrate before loading

- No major infection or soft tissue inflammation should be present. Small periapical granulomas is not a contraindication as they can be eliminated by surgical curetting and subsequent osteotomy

- Sufficient bone apically to ensure primary stability.

In the maxillary esthetic zone, implant placement should be undertaken towards the palatal aspect of the extraction socket and one should not follow the same direction of the extraction socket. Care must be taken during the osteotomy preparation on the palatal wall, as this is dense and difficult to prepare.

If immediate provisional restoration is considered, it is essential to have about $35 \mathrm{Ncm}$ of insertion torque. If moderate primary stability of about $25 \mathrm{~N} \mathrm{~cm}$ is achieved, then a tranmucosal healing screw can be placed to avoid second stage surgery. If however, there is poor primary stability with less than $20 \mathrm{~N} \mathrm{~cm}$, then it is advisable to place cover screws and submerge the implant and wait for osseointegration ( 3 to 4 months) before loading the implant. The purpose of the following case reports is to further explain and elucidate the concept of immediate implantation and when it is not advisable to immediately load.

\section{CASE REPORT 1}

A female aged 40 years, the CE 0 of a multinational company fractured the crown off from her upper right first premolar and was faced with an embarrassing situation because the tooth gap was visible when she smiled. She requested an immediate esthetic solution with a fixed restoration (Figs $1 \mathrm{~A}$ and $B$ ). Looking to the emergency of the situation, the treatment plan was to extract the remaining root and place an implant immediately with nonfunctional loading. This plan according to us would be the most conservative (no reduction of adjacent natural teeth) and long lasting method of rehabilitation. On an emergency basis, the next day; after the usual preoperative investigations and medications, the roots were atraumatically removed using first the no 15 blade to sever the periodontal fibers (Fig. 1C) followed by the periotome (Fig. 1D) and finally using the very thin luxator (Fig. 1E). Only after the root was mobile, was the straight root forceps used to remove the roots (Fig. 1F). There were two separate roots with a clear septa (Fig. 1G). It 


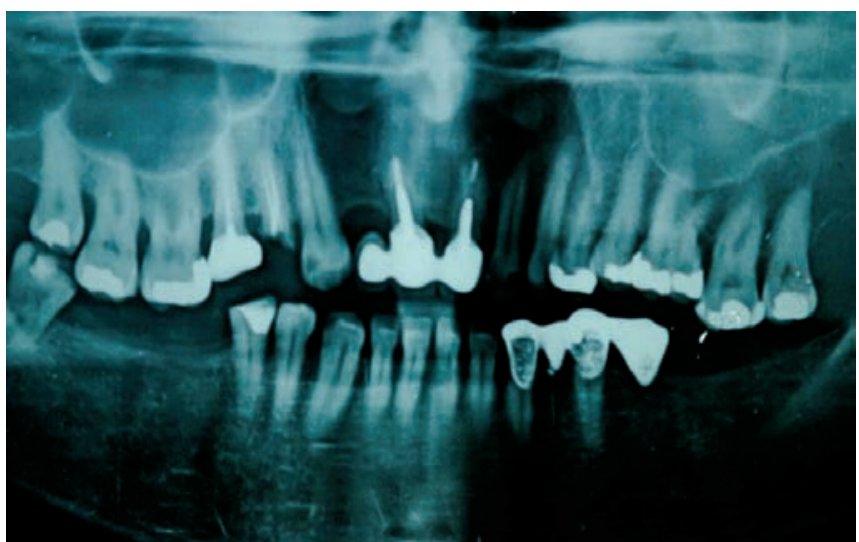

Fig. 1A: Preoperative OPG

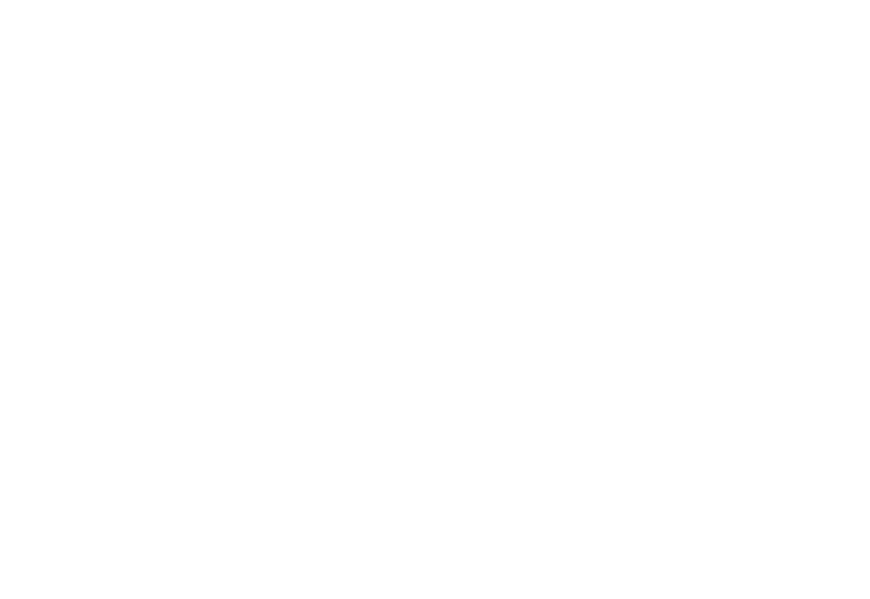

Fig. 1B: Intraoral view of fractured tooth

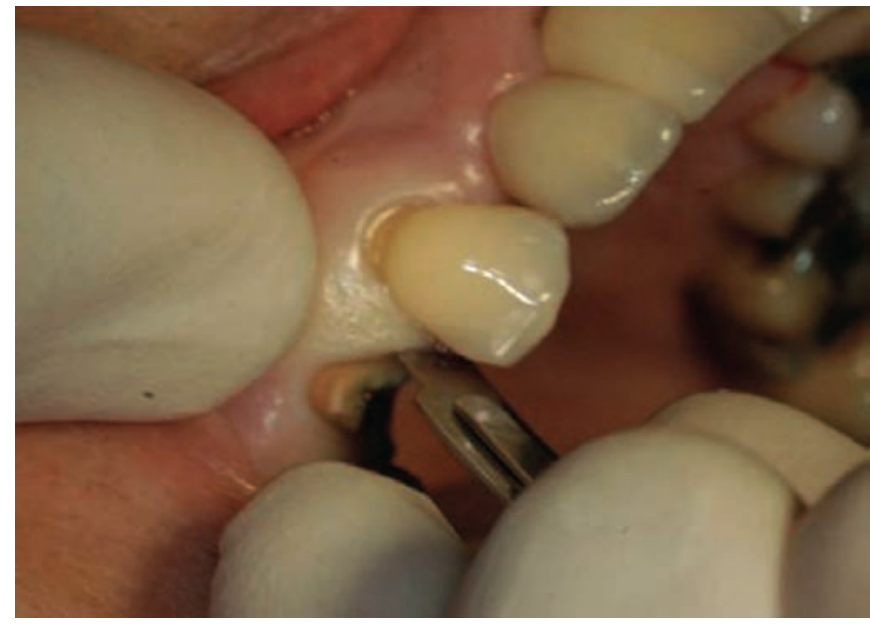

Fig. 1C: Use of no. 15 blade to severe the periodontal fibers

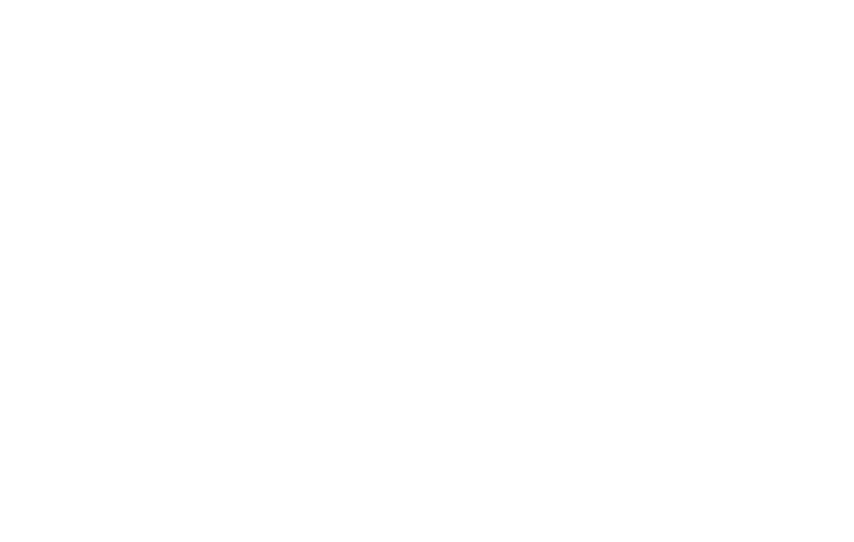

Fig. 1D: Subsequent use of periotome to severe and widen the periodontal membrane space

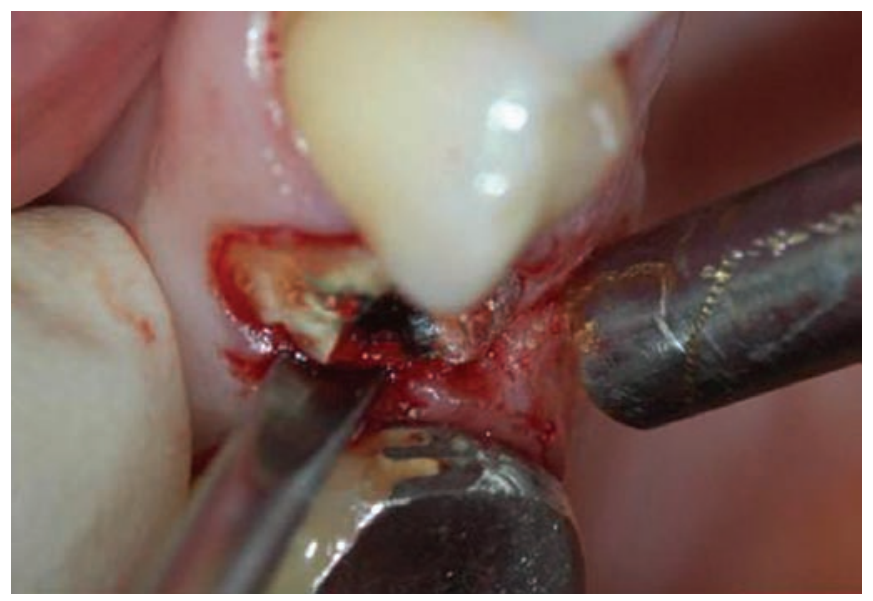

Fig. 1E: Use of luxator on the mesial and distal surface

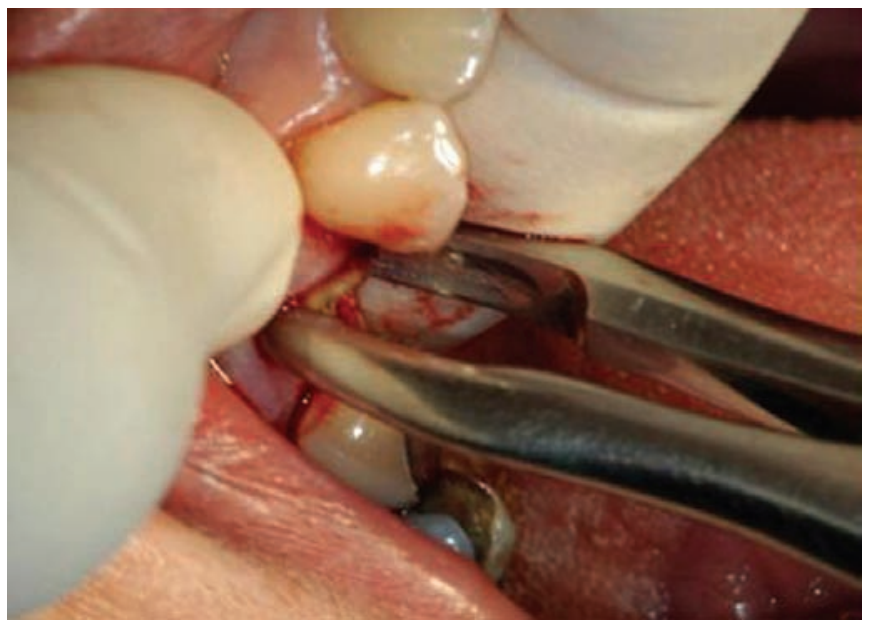

Fig. 1F: Use of forcep only after tooth is mobile 


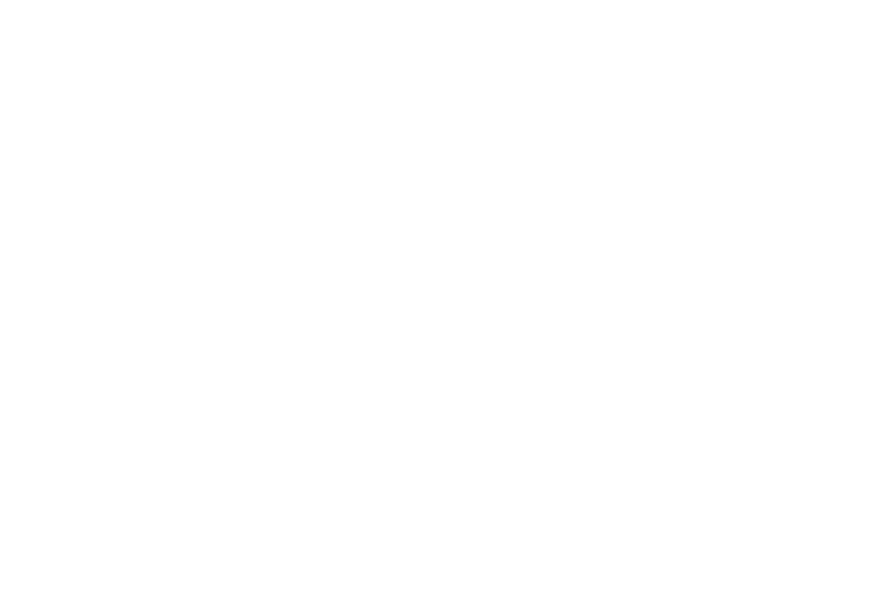

Fig. 1G: Intraoral view of extraction socket

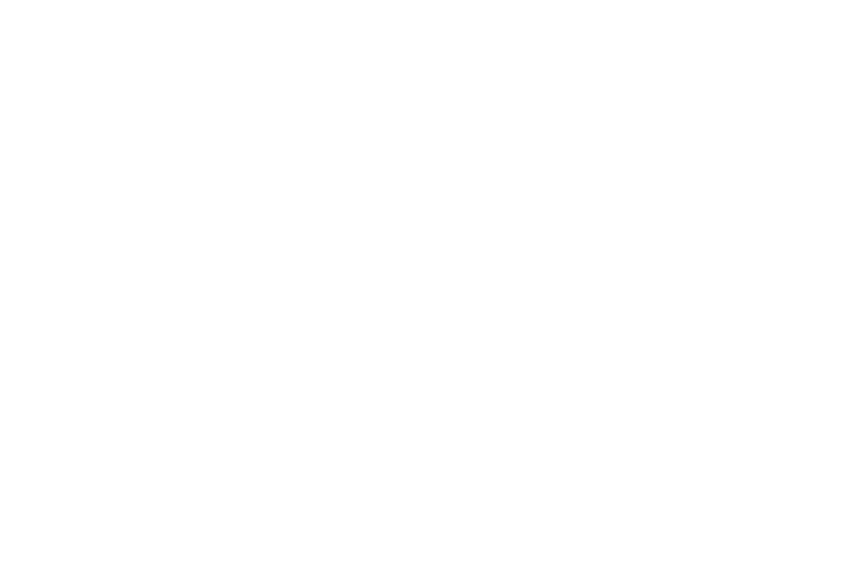

Fig. 1H: Conical reamer prepares the osteotomy to the final shape and more importantly, is an indicator of the primary stability of the implant

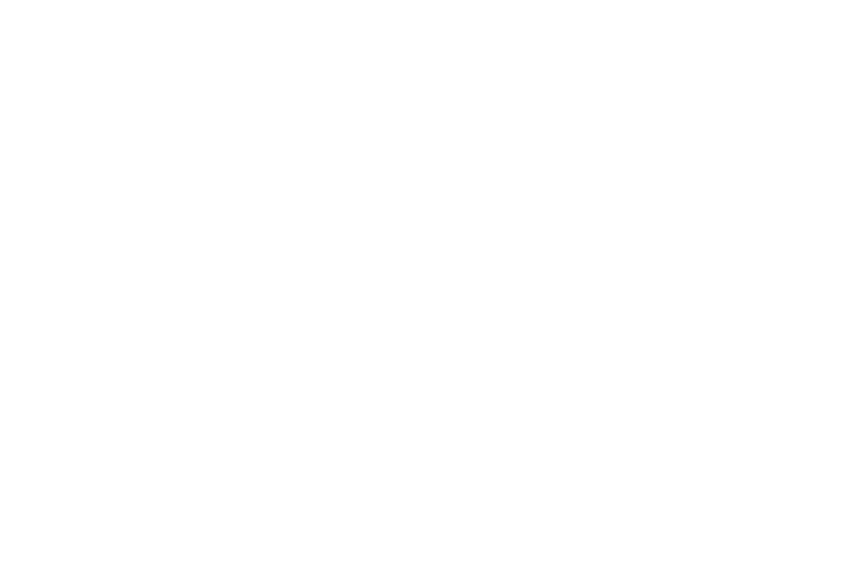

Fig. 1l: Final placement of the implant $3 \mathrm{~mm}$ below the free gingival margin and about $1 \mathrm{~mm}$ subcrestally

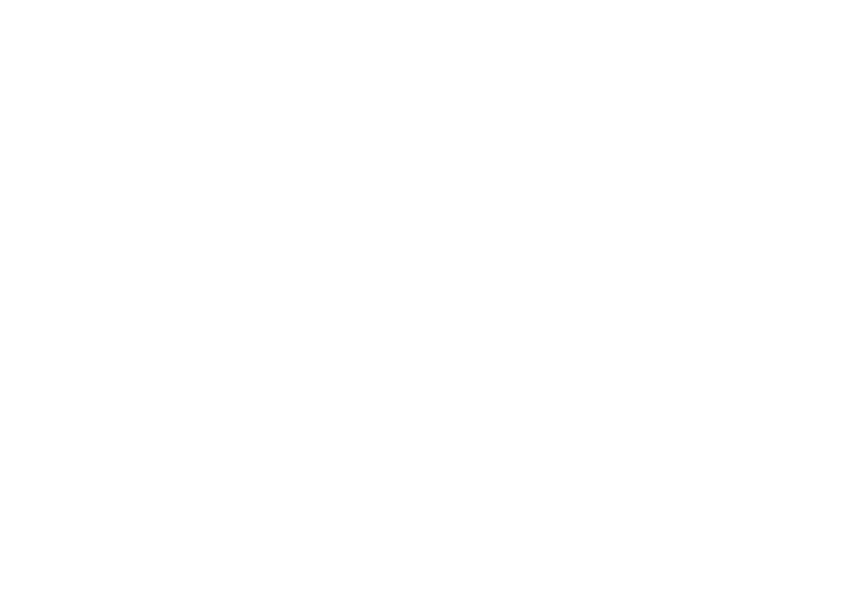

Fig. 1J : Modified abutment torqued in by hand and access hole sealed with temporary elastic composite

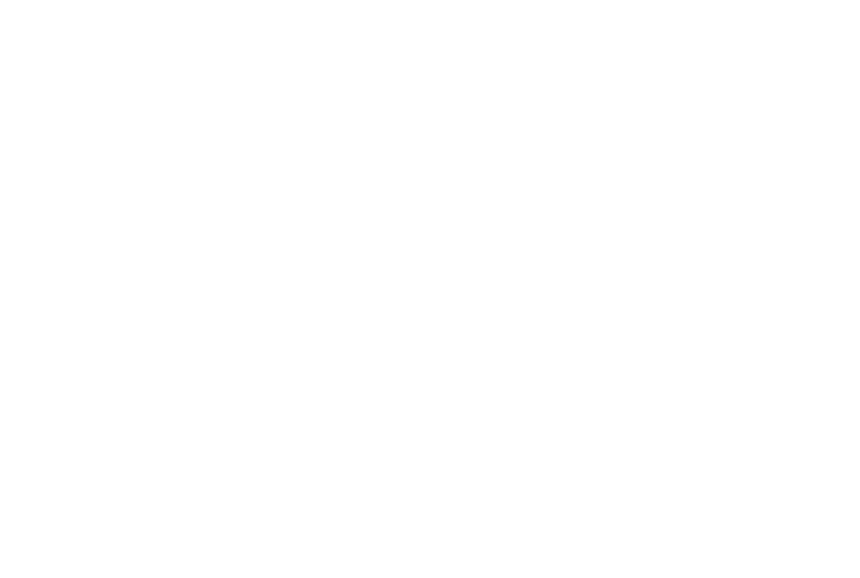

Fig. 1K: Temporary crown cemented with noneugenol temporary cement temporary bond NE (Kerr) at the time of surgery

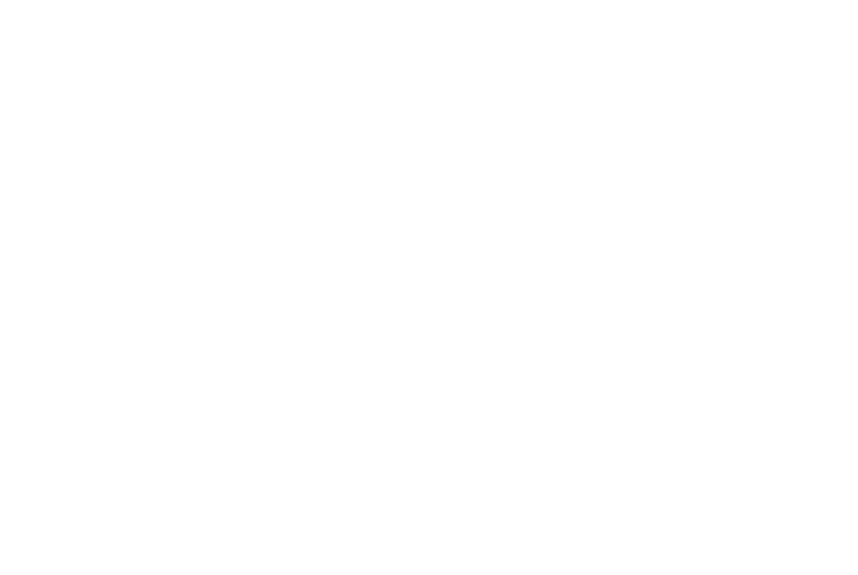

Fig. 1L: Temporary crown after one month without any modification. Note the excellent healing and support of the hard and soft tissues because of the morse taper connection of the "Ankylos" implant system 


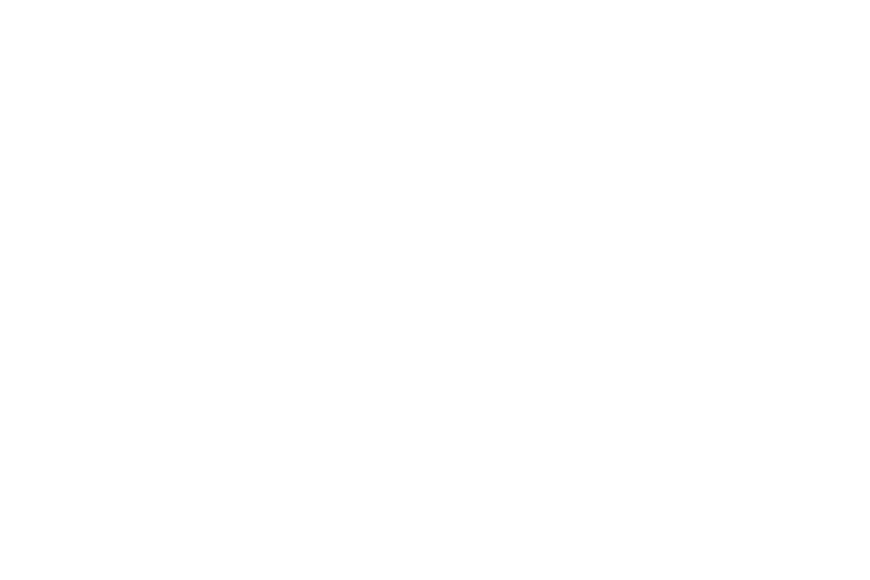

Fig. 1M: Final PFM crown after 3 months postsurgery

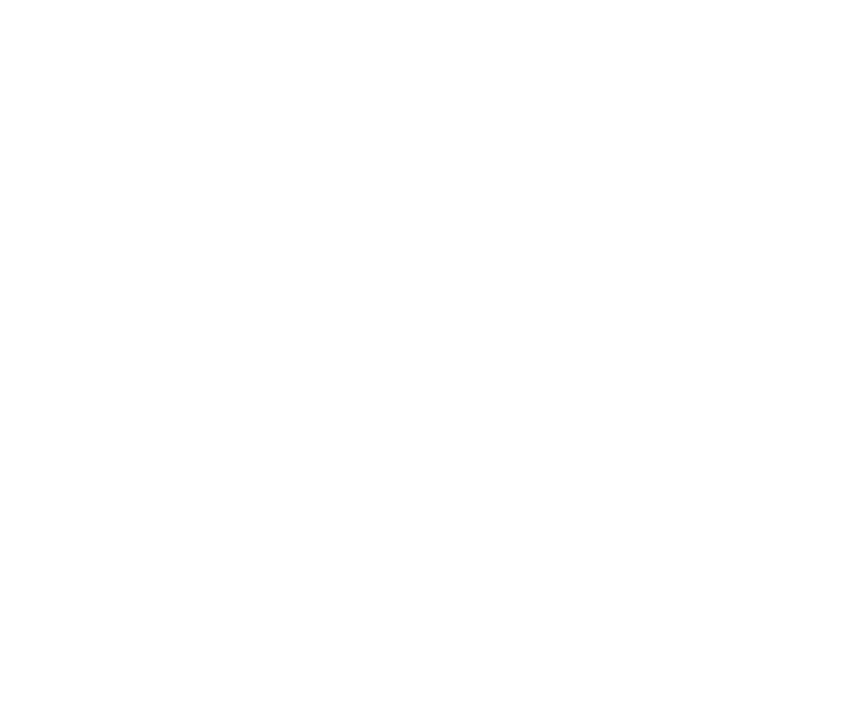

Fig. 1N: Final intraoral radiograph before cementation of crown

was decided to use the 'A nkylos' Implant system because of its establ ished 'tissue care' concept of preserving crestal bone and in turn soft tissue around the implants (Figs $1 \mathrm{H}$ and I). ${ }^{4-7}$

It was also decided to use the 'A nkylos' Balance A butment (Fig. 1J) at the time of surgery itself and modify it extraorally. The advantage of placing the final abutment at the time of surgery, avoids the need to change abutments and in turn preserves the bone and soft tissue., 8,9 The precaution to take is to only torque the abutment with hand pressure at the time of surgery. The final torquing with the wrench should be undertaken after osseointegration, i.e. minimum of two months. With the 'cell plus' surface of the 'Ankylos' implants which is treated right up to the shoulder of the implant, accelerated hard and soft tissue integration is achieved (Figs $1 \mathrm{~K}$ to $\mathrm{N}$ ).
Part 2 of the article will demonstrate with case reports when it is advisable not to load the implants immediately after implantation.

\section{CASE REPORT 2}

Patient aged 60 presented with fractured crowns and requested urgent rehabilitation. Radiological examination revealed no radiolucencies in the periapical region (Fig. 2A ). It was therefore decided to do extraction and immediate implantation. Subsequently the roots were extracted atraumatically using microsurgical blade no. 15, followed by periotomes and fine luxators (Figs $2 \mathrm{~B}$ and $\mathrm{C}$ ). Two 'A nkylos' implants were placed $1 \mathrm{~mm}$ subcrestally (Fig. 2D). The primary stability was only $25 \mathrm{~N} \mathrm{~cm}$ and it was therefore decided not to place provisional restoration. However, the coverscrews were removed and sulcus formers were placed with transmucosal healing, thus avoiding second stage surgery and also reducing treatment time (Figs $2 \mathrm{E}$ and F). The patient was rehabilitated with porcelain fused to metal

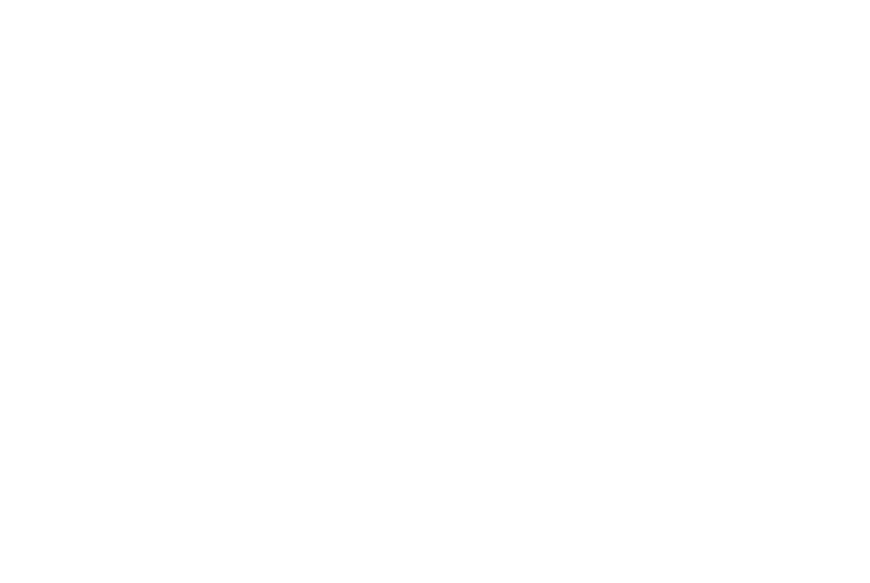

Fig. 2A: Preoperative radiograph

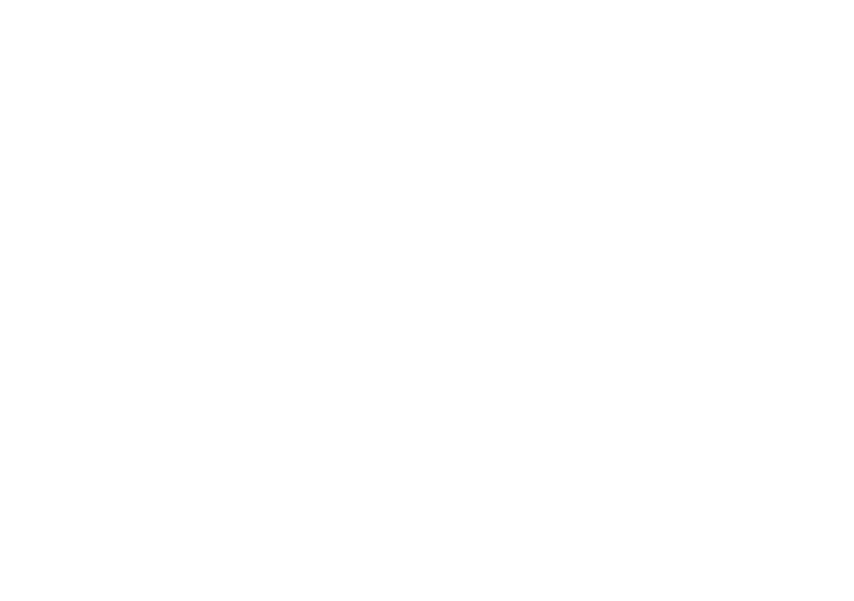

Fig. 2B: Use of microsurgical blade 


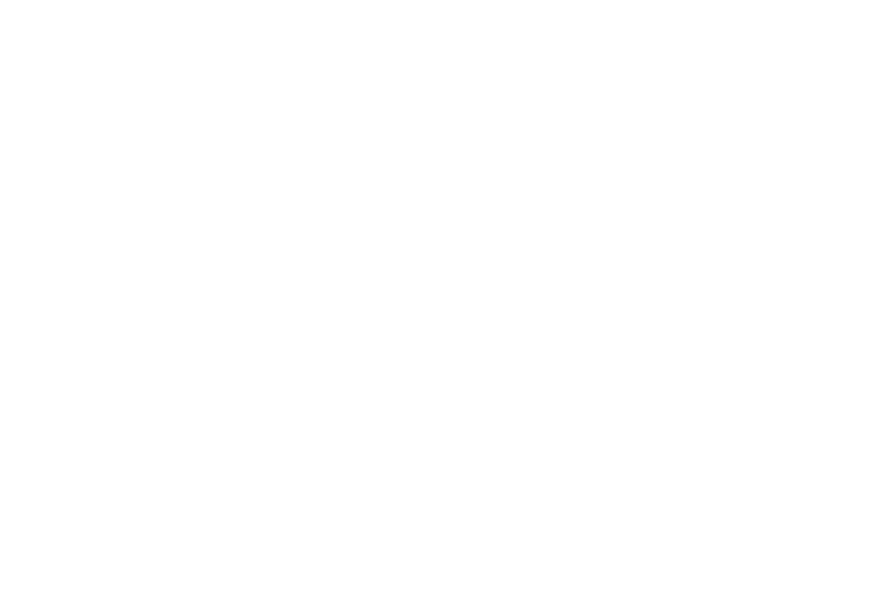

Fig. 2C: Extraction socket

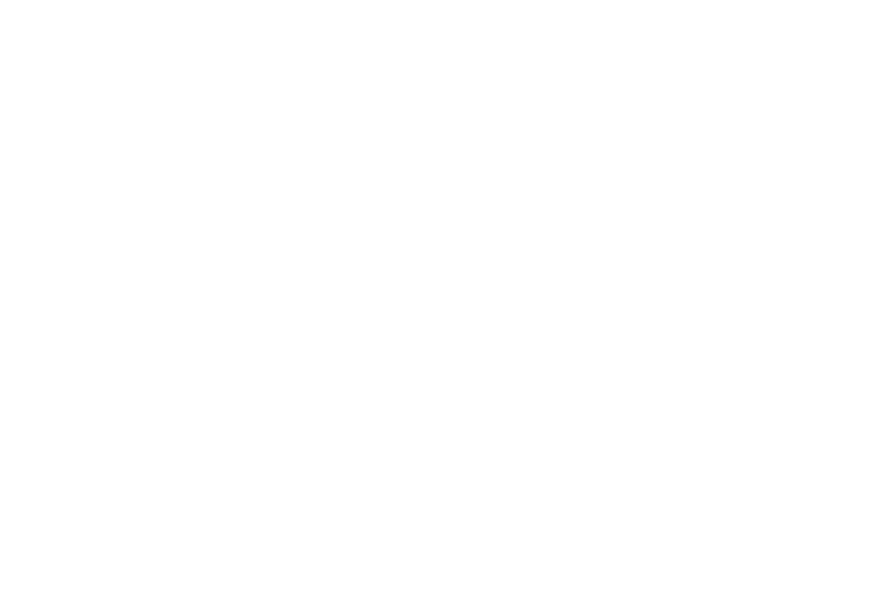

Fig. 2D: Implants placed in the extraction sockets

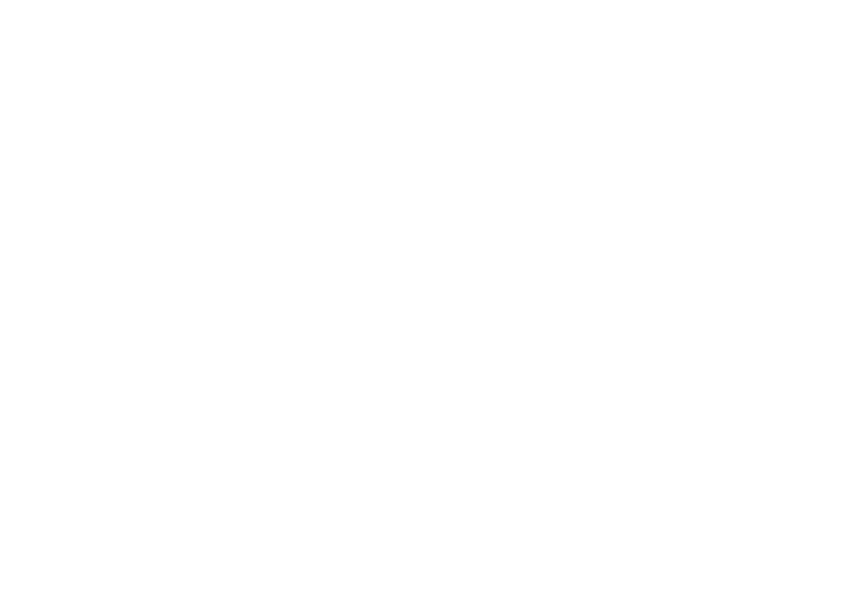

Fig. 2E: Sulcus formers placed

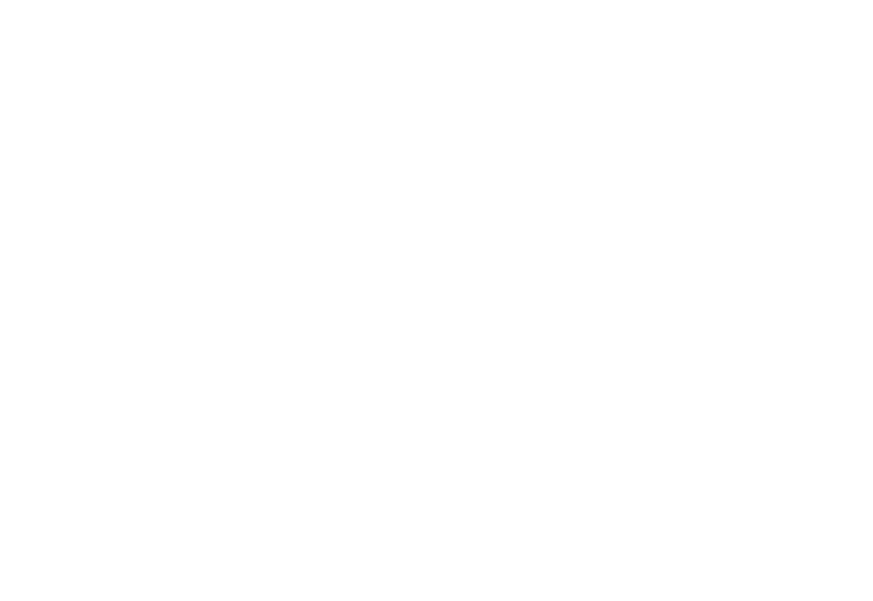

Fig. 2F: Intraoral radiograph following implant placement

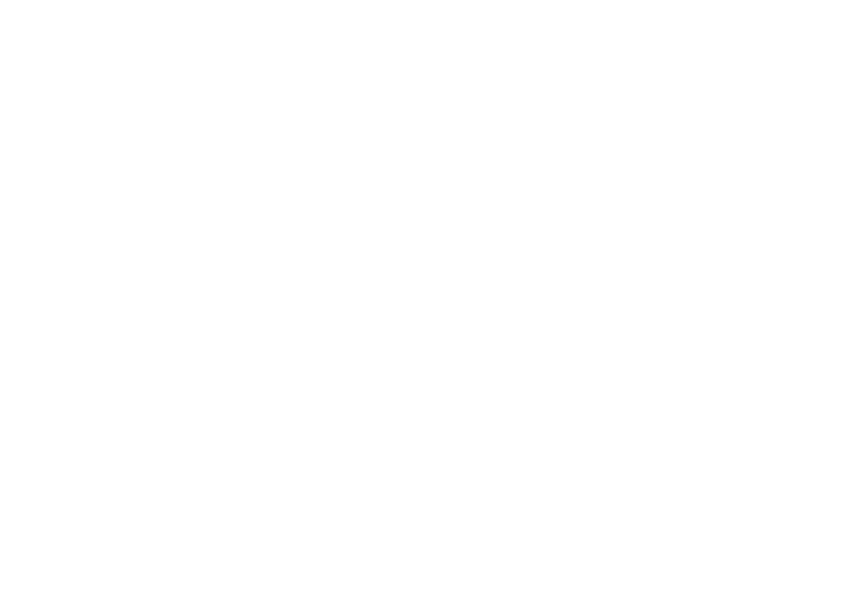

Fig. 2G: Final splinted crowns cemented in place

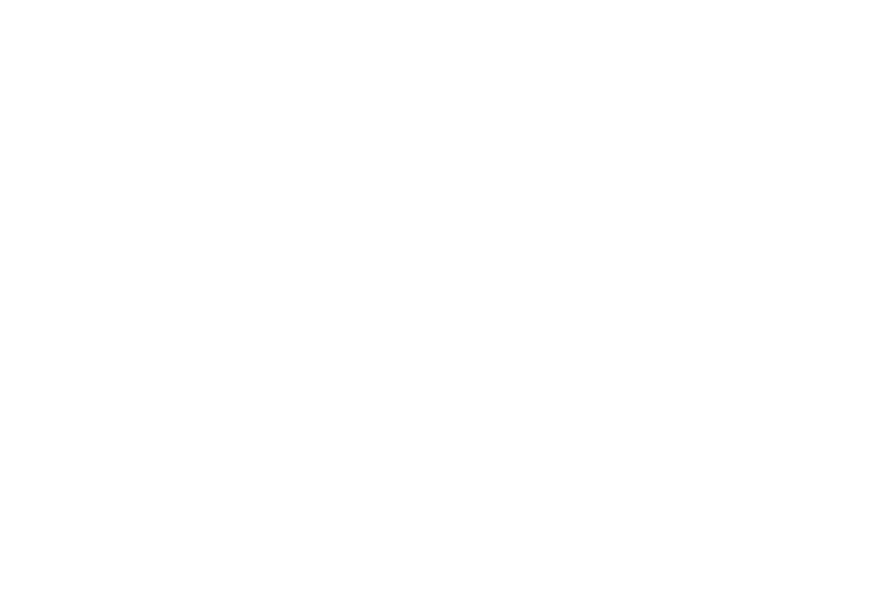

Fig. 2H: Final Intraoral radiograph following cementation of final prosthesis. Note: The excellent marginal fit of the PFM restoration 


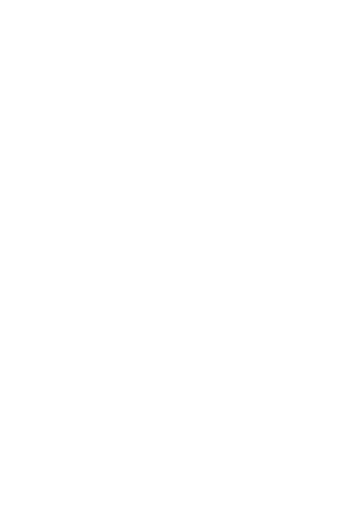

The cervical geometry of threads reduces load transfer to the cortical bone

Continuously increasing thread depths transfers load to the cancellous bone

Fig. 3: Progressive thread design of the 'Ankylos' implant

crowns (PFM) two months after surgery (Figs $2 \mathrm{G}$ and $\mathrm{H}$ ). The reduced treatment time of two months after surgery for rehabilitation was possible due to the grit blasted and thermally acid etched "cell plus" surface of the 'A nkylos' Implant and also due to the patented progressive thread design of the 'A nkylos' implant (Fig. 3).

\section{CASE REPORT 3}

A male aged 42 years reported with fracture of his left lateral incisor which was fractured vertically. Since there was a possibility of loss of labial bone due to pathology, it was decided that we would only extract and wait for 3 weeks for soft tissue closure to take place. As expected after extraction it was found that the labial bone was partially lost in the coronal half of the socket (Figs 4A and B). Therefore, the tooth was only extracted and the socket closed. A modified Maryland bridge of composite was bonded to the palatal surface of the adjacent teeth (Fig. 4C). The plan was to wait for 3 weeks and then implant with simultaneous guided bone regeneration (GBR). A fter 3 weeks of healing (Fig. 4D) it was decided to take a full thickness mucoperiosteal flap with two vertical incisions (Fig. 4E). A fter the usual osteotomy on the palatal aspect of the socket, a $4.5 \mathrm{~mm}$ diameter and $14 \mathrm{~mm}$ long 'A nkylos' implant was placed with excellent primary stability. Cover screw was removed and sulcus former was placed to support soft tissue and facilitate second stage surgery (Fig. 4F). However, immediate provisionalization on the implant was avoided because of the necessity of bone grafting. From the experience of the authors they have found that if the membrane in $G B R$ is exposed then optimum bone generation does not take place. A rtificial bone mineralized Algae (A Igipore, Dentsply Friadent) (Fig. 4G) was placed to cover the exposed threads of the labial surfece. The grafted bone was next covered with a resorbable membrane (BioGide Geistlich, Switzerland) (Fig. 4H). Good primary closure of the wound was achieved due to waiting for 3 weeks and due to design of flap (Fig. 4I). A new modified ' $M$ aryland' bridge was bonded immediately after surgery. Figure 4J show intraoral radiograph taken immediately after surgery.

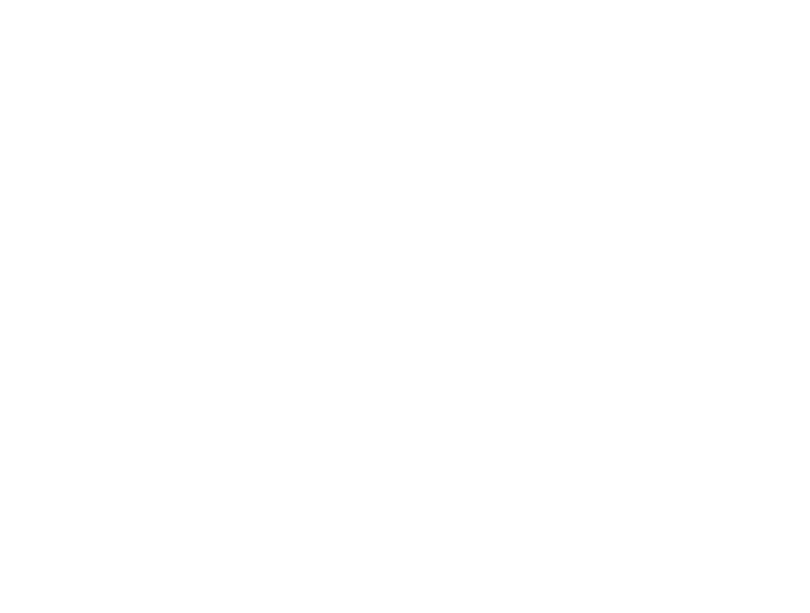

Fig. 4A: Vertically fractured root

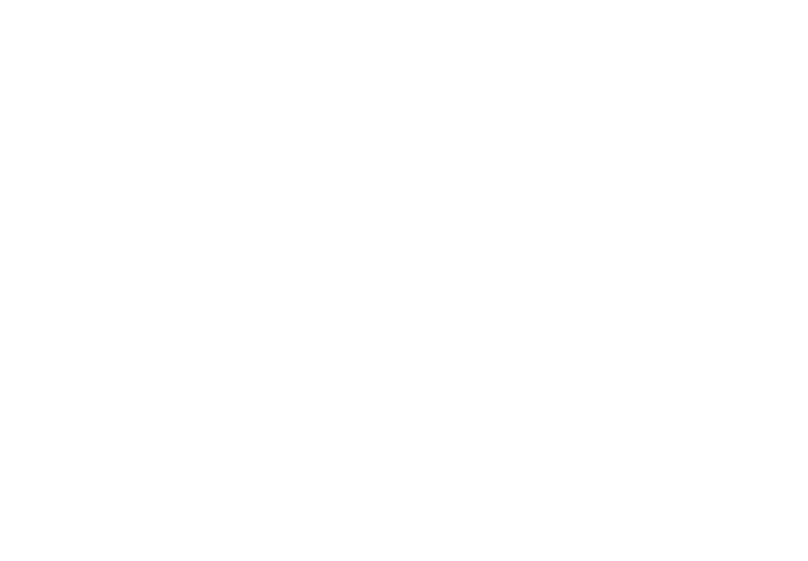

Fig. 4B: Loss of coronal half of labial plate

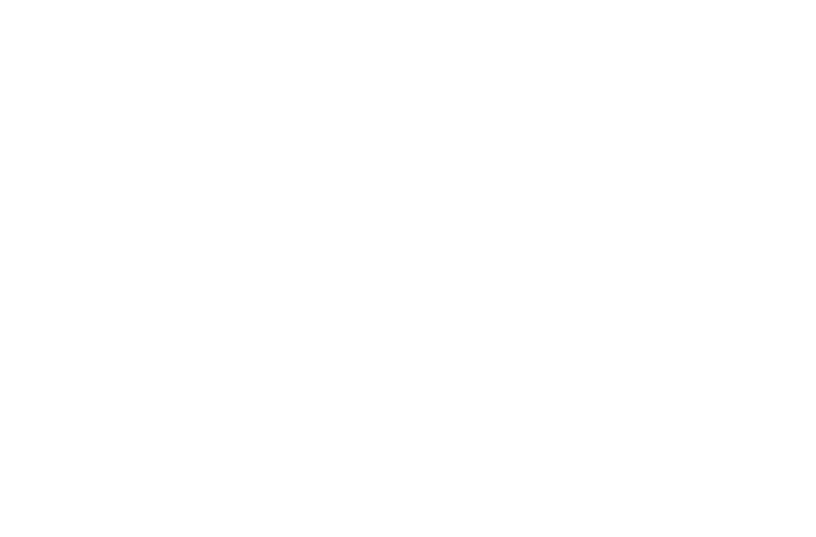

Fig. 4C: Modified "Maryland bridge" bonded to adjacent teeth 


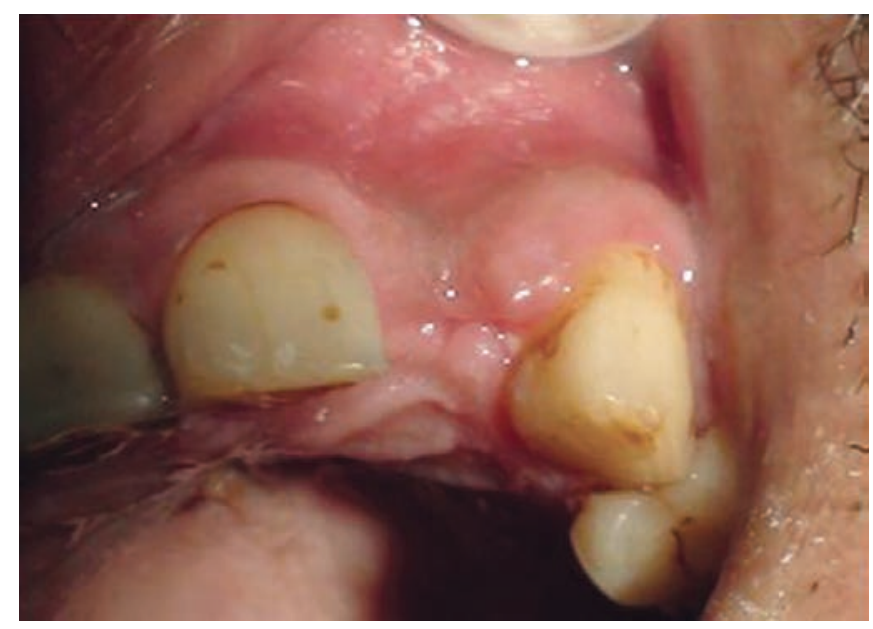

Fig. 4D: After 3 weeks of soft tissue closure

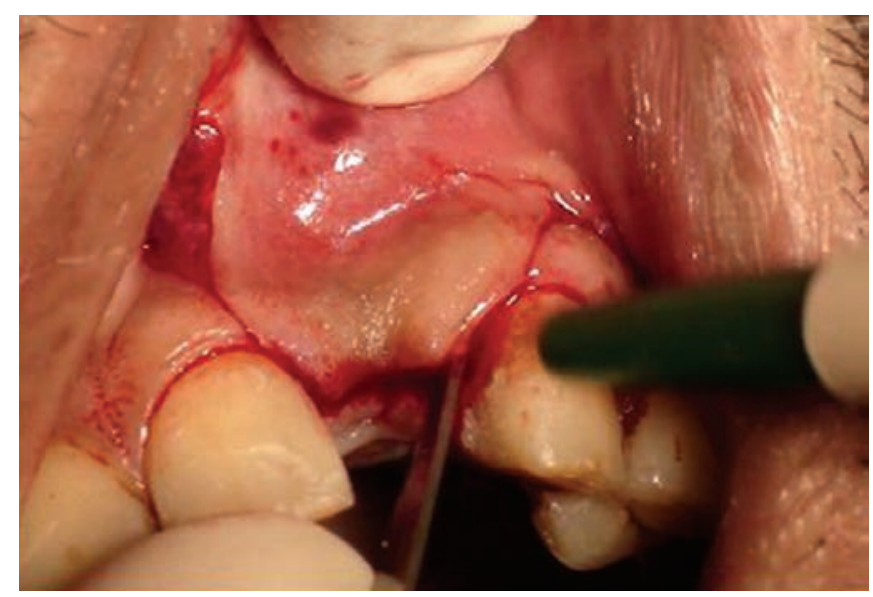

Fig. 4E: Use of microsurgical blade for incision

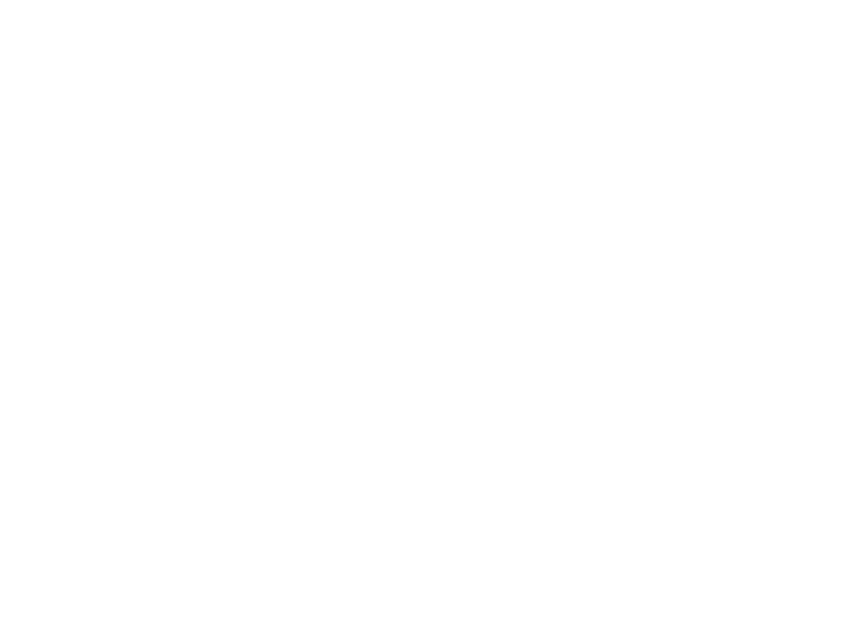

Fig. 4F: Sulcus former placed

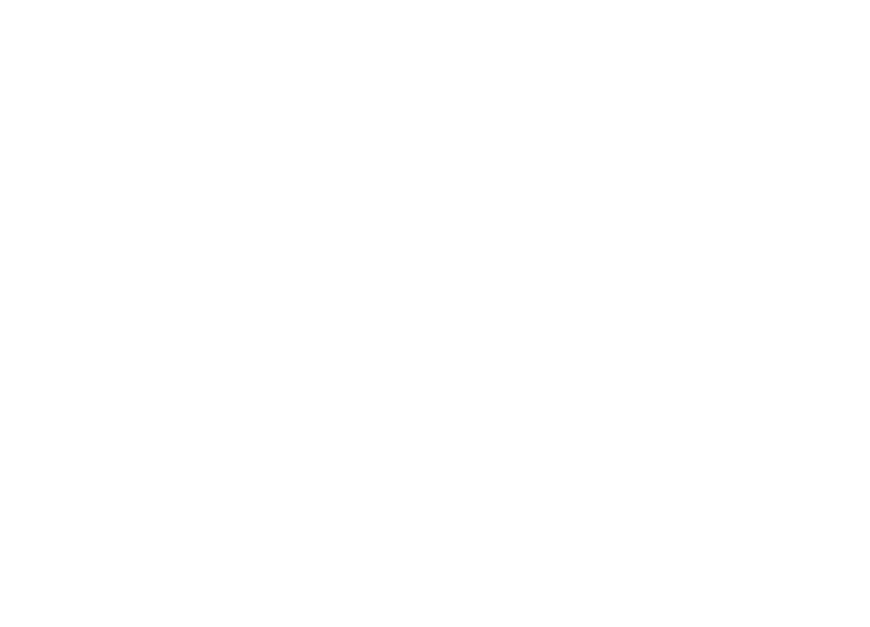

Fig. 4G: Algipore bone graft material placed

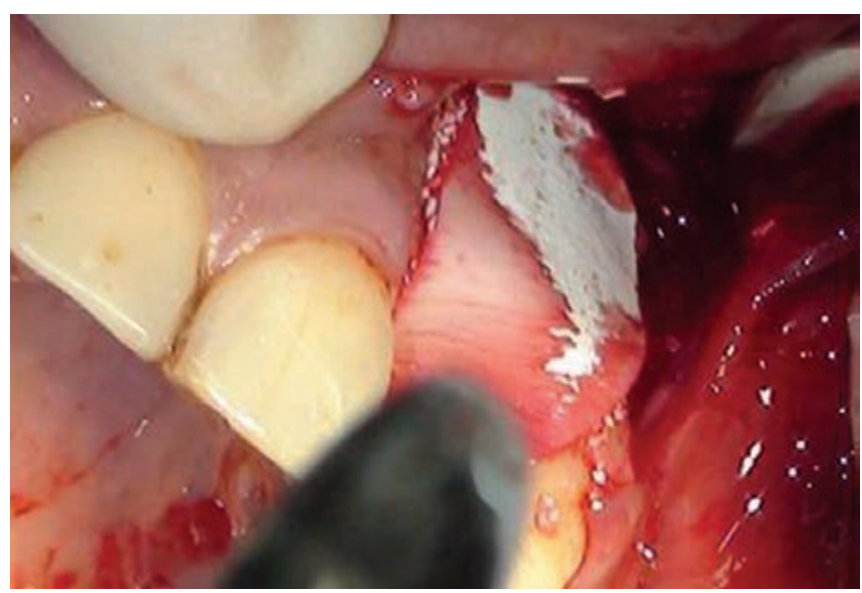

Fig. 4H: Algipore covered by BioGide membrane

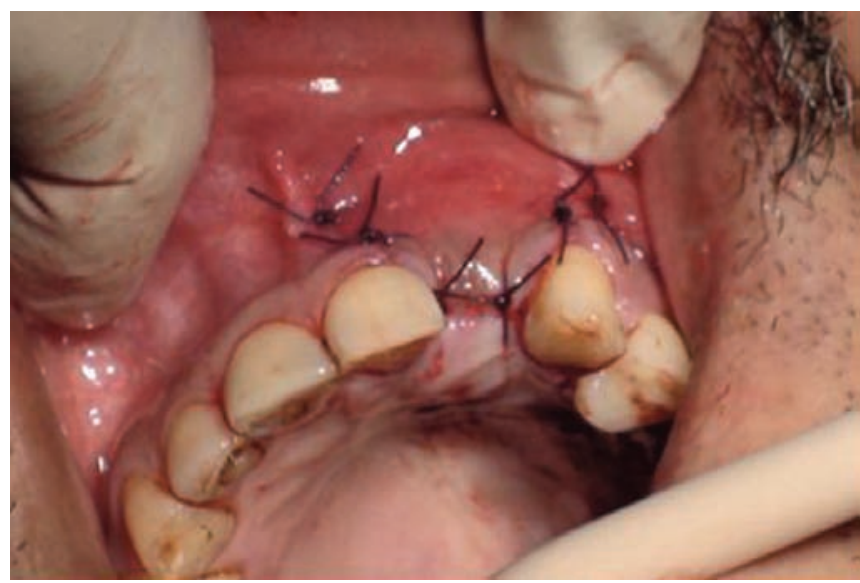

Fig. 4l: Achievement of primary closure due to delayed implant placement 3 weeks after extraction and design of flap 


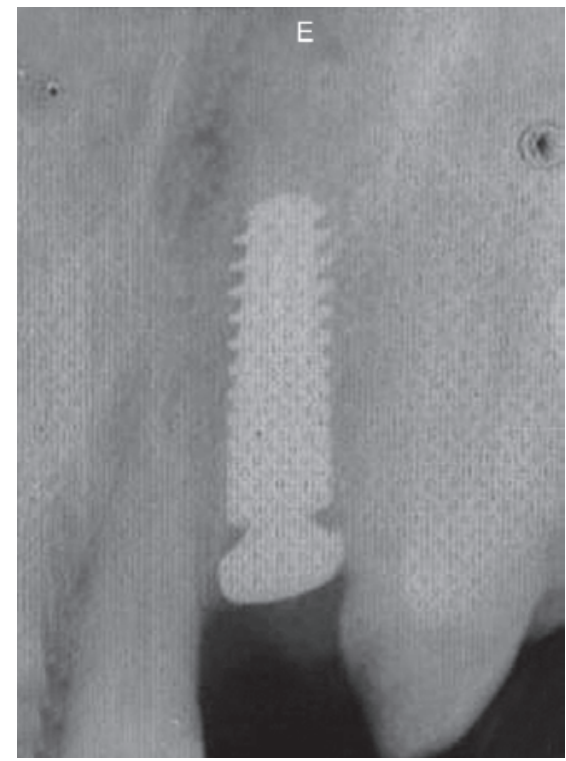

Fig. 4J : Intraoral radiograph taken immediately after surgery

\section{CONCLUSION}

- Although extraction, immediate implant placement and nonfunctional loading is an acceptable procedure, case selection and experience is requi red before undertaking it.

- Implant placement is typically undertaken tow ards palatal aspect of extraction socket.

- Avoid teeth with large or acute periapical infections.

- Avoid teeth with large bone dehiscence or fenestration defects.

- Adequate primary stability of atleast $35 \mathrm{Ncm}$ is required for immediate nonfunctional loading.

- Adequate bone should be present all round the implant covering it.

- Preferably no grafting procedure should be undertaken especially not major grafting. In which case primary closure is indicated and wait for tissue and implant to integrate before loading.

- No major infection or soft tissue inflammation should be present. A small chronic periapical granuloma is not a contraindication because it can be eliminated by surgical curettage and subsequent osteotomy.

- The documented advantages of immediate implantation are: (a) Preservation of hard and soft tissues (b) Psychological benefit to the patient (c) No need to wear removable dentures.

- Reduces total treatment time by more than $50 \%$ and also eliminates need for second stage surgery.

- Tapered progressively threaded implants like the "Ankylos" (Dentsply Friadent) is documented to give best success rates with immediate implantation.

- Grit blasted and thermally acid etched rough implants cell plus (Dentsply Friadent) has been documented with increased surface for bone to implant contact (BIC) and also contributing to improved primary stability.

- Loading should be nonfunctional loading with no occlusal contact in centric and in excursive motions.

- Progressive loading after one and a half months to two months can be started to improve bone density.

- The implant abutment connection should have platform shifting from the periphery to the central axis of the implant. However, more important, it should have a friction locked morse taper conical connection which elimines microgaps and micromovements and in turn preserves hard and soft tissues (A nkylos Dentsply Friadent).

\section{REFERENCES}

1. B ranemark PI. Osseointegration and its experimental background. J Prosth Dent 1983;50;399-410.

2. Gargiulo, et al. Dimensions and relations of the dentinogingival junctional in humans. J Periodontol 1961;32:111-19.

3. Tarnow DP, et al. The effect of inter-implant distance on the Light of inter-implant bone. J Periodontal 1997;68:186-98.

4. M orris HF, O chi S. The influence of implant design, application, and site on clinical performance and crestal bone, a multicenter, multidisciplinary clinical study. Dental ImplantC linical R esearch Group. Implant Dent 1992;1:49-55.

5. W eng D. Different microgap designs and their influence on Peri Implant Bone levels. A bstract EOA Vienna Journal of periodontology 2003;141.

6. N entwig GA. The A nkylos implant system concept and clinical application. J Oral Implantol 2004;30:171-77.

7. Baggi $L$, et al. The influence of implant diameter and length on stress distribution of oseio integrated implants related to crestal bone geometry: A three-dimensional finite element analysis. J Prosthet Dents 2008;100:422-31.

8. A brahamson, et al. The mucosal barrier following abutment dis/ reconnection: A n experimental study in dogs. J Clin Periodontal 1997;24:569-75.

9. Berglundh, et al. The soft tissue barrier at implants and teeth. Clin Oral Implants Res 1991;2:81-90. 